JOURNAL DE PHYSIQUE IV

Colloque C1, supplément au Journal de Physique III, Volume 2, avril 1992

\title{
COMMENT MESURER LINTENSITE ACOUSTIQUE CORRECTEMENT ?
}

\author{
J. DUMAS \\ Brüel \& Kjaer France, 46 rue du Champoreux, BP. 33, F-91541 Mennecy cedex, France
}

\begin{abstract}
Summan:
Acoustic intensity is a physical term well known and now frequently used by research labs and manufacters to qualify sound sources by measuring their sound power and locating their radiating areas. Acoustic intensity is currently measured with a 2 microphones-probe (P-P probe). The calibration of the measuring system has to be done for reliability of measurements. The New IEC standard 1043 defines required technical specifications of the intensitymeasuring system mainlycomposed byaprobe and a frequency analyser. Particularly, pressure - residual intensity index represents the quality of the system. Brüel \& Kjaer has recently released a calibration system allowing complete calibration of the measuring system. Residual intensity may be used for the final calculation of acoustic intensity but many careshasto betaken. A more reliable and simple wayistomeasureacoustic intensity directly with phase matched channels.
\end{abstract}

\section{Introduction:}

Depuis dix ans, la pratique de la mesure de lintensité acoustique s'est développée pour permettre une meilleure approche et connaissance des sources sonores. Elle permet principalement la mesure de la puissance acoustique des sources dans des milieux réels tels que les ateliers. La connaissance des flux d'énergie sonore dans le champ proche des sources permet de repérer les zones actives dans le rayonnement sonore. Les limites des mesures de l'intensité acoustique sontmaintenant connues. Outre celles liéesàl'environnement de mesure (bruits parasites, réverbération de la salle... ), d'autres sont dues au principe même de la méthode. Si la limite reliée à l'approximation du vecteur vitesse particulaire est immuable, celle amenée par linstrumentation peut être réduite considérablement en prenant les précautions nécessaires. La norme d'instrumentation CEl 1043 propose les performances à respecter. Le respect de ces critères passe par la validation de lintensimètre qui peut être réalisé par le système d'étalonnage Brüel \& Kjaer Type 3541. Le fonctionnement de ce système s'avérant extrêmement simple et logique, il peut être pratiqué sur le site de mesure, pour assurer la flabilité des résultats. La notion de l'intensité résiduelle propre à chaque intensimètre doit être obligatoirement connue pour connaître ses limites d'utilisation. De plus, lorsque peu d'attention est portée sur linstrumentation, l'intensité résiduelle est très variable dans le temps, ce qui rend impossible l'obtention correcte des niveaux d'intensité acoustique surtout si des fichiers de correction sont utilisés pour le résultat final. Il apparaf́t alors, en pratique, beaucoup plus facile d'utiliser un intensimètre apparié en phase doncòniveau d'intensité résiduelle faible et.quiplus est, garantistable dans le temps.

\section{Lamesure de ilintensité acourtique:}

La mesure de ce paramètre a été déjà largement abordée dans la littérature. La figure 1 résume le principe de l'estimation de lintensité acoustique.

Pour des signaux sinusoïdaux, lintensité acoustique intégrée temporellement s'exprime (1) :

(1) $\quad I r=\frac{P_{A} P_{B} \cdot \sin \phi}{\omega \rho \Delta r}$

$$
\text { où } \begin{aligned}
\omega & =\text { fréquence angulaire }=2 \pi F \\
\phi & =\text { phase mutuelle de } P_{A} \text { et } P_{B}
\end{aligned}
$$




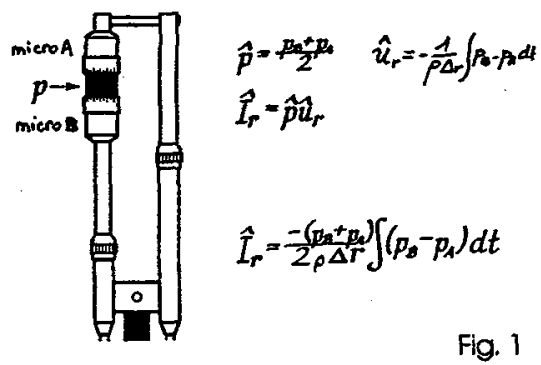

\section{L'étalonnage de l'intensimètre:}

L'équation (1) montre que le résultat final de la mesure de l'intensité acoustique surune direction rest fonction des deux pressions acoustiques mesurées, de leurphasemutuelle, de lafréquencéangulaire, de la masse volumique et enfin de l'écartement entre les microphones. Tous ces paramètres doivent être maîtrisés. La complexité de la formulation de lintensité acoustique rend difficile l'étalonnage des moyens de mesures. La maittrise de chaque paramètre se fera comme suit.

- La masse volumique $\rho$ : En général, elle est calculée à partir de termes physiques mesurables tels que la pression athmosphérique et la température.

(2)

$$
\rho=0.348534 \frac{P_{\text {ann }}}{273+T}
$$

$$
\begin{aligned}
& \text { où } \quad P_{\text {olm }}=\text { pression atmosphérique en } \mathrm{hPa} \\
& T \stackrel{\text { atrn }}{=} \text { Température en }{ }^{\circ} \mathrm{C}
\end{aligned}
$$

Les paramètres $P_{\text {atm }}$ et T sont rentrés numériquement dans l'analyseur. $\rho$ est exprimé en $\mathrm{kg} / \mathrm{m}^{3}$.

- L'écartement des microphones $\Delta r$ : ll est défini par la configuration de la sonde. Des essais de laboratoire ont permis une optimisation de cette configuration et donc la stabilité de $\Delta r$ en fonction de la fréquence. La valeur est rentrée numériquement dans lanalyseur. Elle entraine une limite haute fréquence liée au principe de l'approximation du gradient de pression.

- Les pressions acoustiques $P_{A}$ ef $P_{B}$ : Chacun des microphones pourra être soumis à un étalonnage classique par pistonphoneou source sonoréétálon tel que courammentpratiqué pourlessonomètres. L'intérêt de cetteaction est d'avoir apparié en amplitude la sensibilité des deux voies de mesure.

- La fréquence angulaire : Elle est intrinsèque au phénomène sonore à mesurer. Elle est toutefois dépendante dans le résultat, de la résolution en fréquence de la méthode de mesure (FFT, filtrage numérique ou analogique).

- La phase mutuelle des signaux de pression $P_{A}$ et $P_{B}$ : Cette quantité se révèle comme très importante dans l'estimation finale de lintensité acoustique en basse fréquence car le moindre désappariement en phase des voies de mesure entraîne une erreur. Elle se caractérise par l'apparition d'une intensité résiduelle.Un biais de quelques dizaines de degrés donne une intensité résiduelle importante. L'intensité résiduelle est intrinsèque à l'instrumentation et ne caractérise pas le champ sonore au point de mesure. Elle se mesure très facilement par simulation d'un champsonore d'intensité acoustiquenulle. Elle définit la limite bassefréquencedel'instrumentation.

\section{Lanorme d'instrumentation CEL 1043 ;}

Elle définit les caractéristiques et les grandeurs intenvenant dans la mesure de lintensité acoustique. Egalement, lesspécifications des moyens de mesure son imposées et classifiées en 2 classes principales (classe l et2). L'indice de pression - intensité résiduelle minimum s'avère l'élément principal de caractérisation de lintensimètre et de distinction des classes. L'intensité résiduelle est produite intrinsèquement parle désappariementen phase (eneur de phase) des 2 voies de mesure de l'intensimètre (sonde + analyseur). L'équation (3) la définit comme suit :

(3)

$$
\mathrm{I}_{\mathrm{res}}=-\frac{\mathrm{P}_{\mathrm{o}}^{2}}{\rho \mathrm{c}} \frac{1}{\mathrm{k}} \frac{\Delta \phi \mathrm{e}}{\Delta \mathrm{r}}
$$

où $l_{\text {res }}=$ intensité résiduelle $\left(\mathrm{w} / \mathrm{m}^{2}\right)$

$\mathrm{P}_{\mathrm{O}}=$ pression acoustique du champ sonore d'intensité active nulle $(\mathrm{Pa})$

$\rho \mathrm{C}=$ impédance acoustique (rayls)

$k=$ nombre d'onde (rad $/ \mathrm{m})$

$\Delta \phi e=$ erreur de phase (rad)

$\Delta \mathrm{r}=$ écartement des microphones $(\mathrm{m})$

L'indice pression - intensité résiduelle s'exprime aussi : 


$$
\delta \rho_{\mathrm{lO}}=\mathrm{L}_{\mathrm{pO}}-\mathrm{L}_{\mathrm{o}}=10 \log \left(\frac{\mathrm{k} \Delta \mathrm{r}}{\Delta \phi_{\theta}}\right)(\mathrm{dB})
$$

$\delta \rho_{\mathrm{p}}$ s'appelle encore écart de champ résiduel. II se mesure dans un champ sonore d'intensité active nulle simulé ou recréé artificiellement par positionnement de la sonde.

Les figures 2 et 3 présentent les exigences de la norme pour l'instrumentation utilisée avec les écartements de microphones de 12 ef $50 \mathrm{~mm}$ (indice pression-intensité résiduelle en fonction de la fréquence).
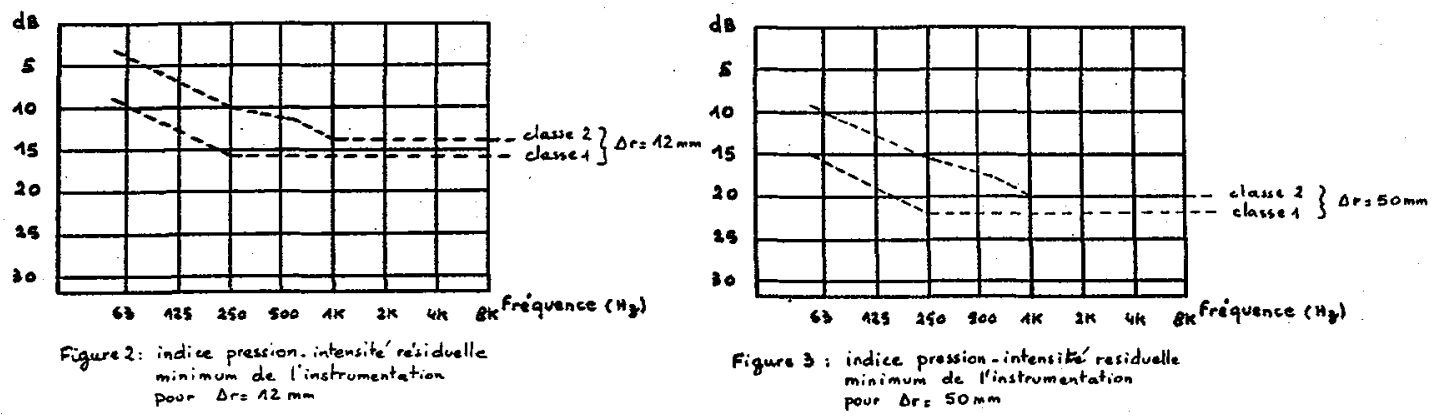

Figure 3 : indice pression-intensité residuelle minimum de I'instrumentation powe $\Delta r=50 \mathrm{~mm}$

\section{La pratique de l'étalonnage de lintensimètre:}

Le système d'étalonnage de lintensité acoustique Brüel \& Kjaertype 3541 est un ensemble compact permettant la vérification complète des systèmes de mesure de pression, d'intensité acoustique et de vitesse particulaire. Le coupleur d'infensité est un moyen original pour simuler le positionnement de la sonde à 2 microphones pour 0 et 90 degrés d'incidence en condition de champ libre. La figure 4 en montre une coupe.

Un pistonphone réalise des étalonnages individuels en niveau de pression, d'intensité et de vitesse particulaire.

Une source sonore large bande (bruit blanc/ bruit rose) assure la mesure de lindice pression - intensité résiduelle. Ceci rend possible la quantification de l'intensité résiduelle durant les mesures.La procédure de calibrage est simple. Les instructions et détails des termes de correction sont donnés dansla fiche d'étalonnage foumieaveclesystème.

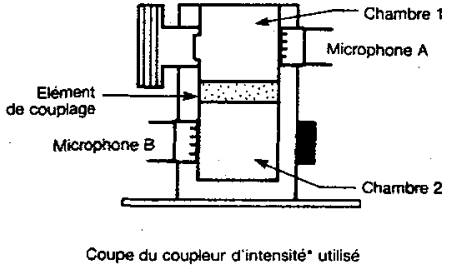

Fig. 4
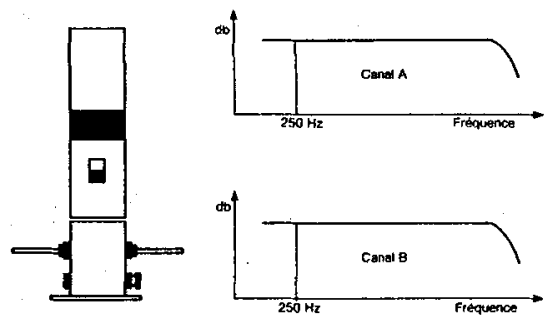

Fig. 5

L'étalonnage en pression (Fig. 5). Un niveau de pression connu, prodult par le pistonphone, foumi un point de référence qui permet de s'assurer que les voies de mesure ont la même réponse. Jusqu'à maintenant, le calibrage d'un équipement de mesure acoustique s'arrêtait là. Un système de mesure d'intensité acoustique requiert d'autres étapes pour garantir une confiance suffisante.

Vérification de mesure de lintensilé acoustique el de la vitesse particulaire (Fig . 6). Lintensité est mesurée à partir des signaux de pression et de vitesse particulaire. La vitesse particulaire est détectée par intégration temporelle du gradient de pression entre les 2 microphones de la sonde. Lors de l'étalonnage en pression, le signal de gradient de pression est égal à 0 . L.e fonctionnement du détecteur de vitesseparticulaire doit être vérifié parl'utilisation du coupleur et du pistonphone en simulant une onde plane incidente à $0^{\circ}$ par rapport à la sonde.
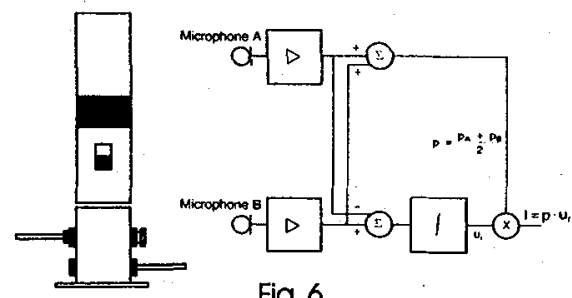

Fig. 6 


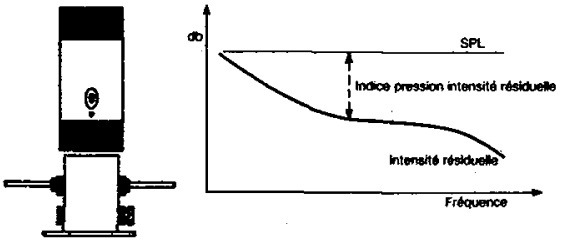

Fig. 7
Mesure de lindice pression -intensité résiduelle (Fig. 7). Quand les sondes d'intensité sont exposées à une onde plane incidente à 90 degrés, les equipements de mesure devralent indiquer un niveau d'intensité égal à $0 \mathrm{w} / \mathrm{m}^{2}$. Ils montreront en fait un niveau d'intensité très inférieur au niveau de pression sonore. Tous les équipements de mesure de lintensité acoustique montreront cet effet, connu sous le nom dintensité résiduelle, qui est un problème valable pour tous les angles d'incidence. En utilisant le coupleur ef la source sonore large bande, on déterminera le spectre dindice pression - intensité résiduelle de lintensimètre. Des microphones à correcteurs de phase permettent d'atteindre des performances ecxellentes. Ils équipent dorénavant toutes les sondes Brüel \& Kjaer et répondent aux exigences de la norme CEI 1043.

\section{Variotions de lindice pression intensité résiduelle: stabilité de lintensimètre:}

A partir de lin dice pression intensité résiduelle, est définie la capacité dynamique de lintensimètre (5) dépendant du niveau de précision requis. La récente nome internationale ISO/DIS 96 14-1 concernant le mesurage de la puissance acoustique par intensimètrie montre l'utilisation de la capacité dynamique dans la validation des résultats de mesure.

(5) $\quad \mathrm{Ld}=\delta_{\mathrm{pOO}}-\mathrm{K}$

(dB) où

Ld = capacité dynamique

$\delta_{p o}=$ indice de pression - intensitè résiduelle $=L_{p o}-L_{0}$

$K=$ facteur définissant la classe de précision

L'intensité résiduelle peut être utilisée pour optimiser lintensité mesurée et obtenir une valeur corrigée.

(6)

$$
l_{c o r}=I_{m}-\frac{P_{m}{ }^{2}}{P_{0}^{2}} \cdot I_{0}
$$

où $\mathrm{I}_{\text {cor }}=$ intensité active corrigé $\left(\mathrm{w} / \mathrm{m}^{2}\right)$

$I_{m}=$ intensité mesurée $\left(w / m^{2}\right)$

$\mathrm{I}_{\mathrm{o}}^{m}=$ intensité résiduelle mesurée dans le coupleur du $3541\left(\mathrm{~W} / \mathrm{m}^{2}\right)$

$P_{m}=$ pression efficace moyenne mesurée $(P a)$

$P_{\circ}^{m}=$ pression efficace moyenne mesurée dans le coupleur du $3541(\mathrm{~Pa})$

L'utilisation de cette formule oblige à garantir la stabilité dans le temps de $\delta_{\mathrm{po}}$. Or, il s'avère que pour différentes configurations électroniques de la chaine de mesurage (gains, filtres de conditionnement...) et parce que tout sejoveà quelques dizièmes de degrés, laformule (6) est trèssouvent inexploitable pour assurerle sérieux du résultat. De plus, la mesure de lintensité acoustique dans les champs réactifs renforce l'exigence de stabilité.

Evidemment, si le constructeur de l'instrumentation assure des valeurs minimum pour $\delta_{\mathrm{pp}}$, alors cette formule de correction est utilisable. Brüel \& Kjaer garantit dorénavant pour toute combinaison sonde/analyseur de fréquence, une erreur de phase entre voles de mesure de $0,15^{\circ}$ maximum de $20 a ̀ 250 \mathrm{~Hz}$. Dans ce cas, laformule (6) ne joue que sur quelques centièmes de $\mathrm{dB}$. Une mesure régulière de $\delta_{\mathrm{po}}$ assure la mantrise de lintensimètre ef permet de repousser les limites d'utilisation surtout en basse fréquence.

\section{Conclusion:}

Pour mesurer lintensité acoustique correctement, il est absolument nécessaire, outre les précautions d'usage propres aux mesures physiques, de définir lindice pression - intensité résiduelle (écart de champ résiduel) caractérisant la qualité de l'intensimètre. De plus, cet intensimètre devra répondre aux exigences définies par la norme CEI 1043. Unsystèmed'étalonnage pratiqueet simplepermettra demaitriserl'intensimètreàn'importequel moment et d'éviter la pratique de fichiers de correction pour obtenir une valeur fiable. La sensibilité aux erreurs de phase de lintensimètre est trop grande pour pouvoir négliger la stabilité des performances. C'est pourquoi, il est plus sûr de mesurer lintensité acoustique correctement avec un système dont les voies de mesure sont appariès en phase.

\section{Réfërences:}

- CEl 1043 : "instruments For the Measurement of Sound Intensity", 2nd Committee Draft, January 1991.

- ISO 9614-1 : "Détermination des niveaux de puissance acoustique des sources sonores par intensimètrie - Mesurage par points".

- "Système d'étalonnage pour les appareils de mesure de lintensité acoustique. Un outil au service des industriels" Jacky DUMAS, Brüel \& Kjaer France. Congrès International de Métrologie, 17, 18 et 19 septembre 1991, Lyon.

- Manuel d'instruction du système d'étalonnage d'intensité Brüel \& Kjaer type 3541, BE 1024-11. 